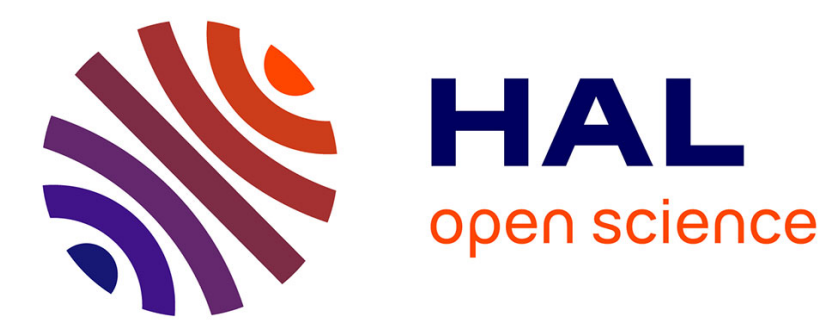

\title{
Broadband coupling transducers for magneto-inductive cables
}

\author{
R R A Syms, L Solymar, I R Young
}

\section{To cite this version:}

R R A Syms, L Solymar, I R Young. Broadband coupling transducers for magneto-inductive cables. Journal of Physics D: Applied Physics, 2010, 43 (28), pp.285003. 10.1088/0022-3727/43/28/285003 . hal-00569648

\section{HAL Id: hal-00569648 \\ https://hal.science/hal-00569648}

Submitted on 25 Feb 2011

HAL is a multi-disciplinary open access archive for the deposit and dissemination of scientific research documents, whether they are published or not. The documents may come from teaching and research institutions in France or abroad, or from public or private research centers.
L'archive ouverte pluridisciplinaire HAL, est destinée au dépôt et à la diffusion de documents scientifiques de niveau recherche, publiés ou non, émanant des établissements d'enseignement et de recherche français ou étrangers, des laboratoires publics ou privés. 


\title{
Broad-band Coupling Transducers for Magneto-Inductive Cables
}

\author{
R.R.A.Syms*, L.Solymar, I.R.Young
}

\section{$\underline{\text { Abstract }}$}

A broadband resonant transducer capable of low-loss coupling between magnetoinductive waveguides and a real impedance is introduced. The transducer is an L-C circuit resonating at the resonant frequency of the elements forming the guide. However, the values of the components in the transducer are different, and chosen to obtain two separate nulls in reflection so that low reflection is obtained over a wide spectral range. The transducer can be incorporated into the MI waveguide itself, allowing a connection between a magneto-inductive cable and a conventional system to be made as a simple splice. The design is confirmed using two metre lengths of low-loss thin-film magnetoinductive cables formed using copper-clad polyimide and operating near $100 \mathrm{MHz}$ frequency.

KEYWORDS Metamaterial, periodic structure, magneto-inductive wave PACS numbers $\quad$ 41.20.-q Applied classical electromagnetism

81.05.Zx New materials: theory, design and fabrication 84.30.Bv Circuit theory 
Optical and Semiconductor Devices Group, EEE Dept., Imperial College London, Exhibition Road, London SW7 2AZ, UK

*Corresponding Author: TEL +44-207-594-6203 FAX +44-207-594-6308 Email r.syms@imperial.ac.uk 


\section{1. $\quad$ Introduction}

Magneto-inductive (MI) waveguides are periodic low-frequency electrical structures, formed by magnetically coupling a set of lumped-element L-C circuits [1]. The properties of linear arrangements and 2D and 3D MI arrays have been extensively studied [2-6]. Propagation losses have been reduced [7], and the effects of non-nearest neighbour interactions [8], bi-periodicity [9], coupling to electromagnetic waves [10] and retardation [11] have all been considered. Although the first demonstrations used simple resonant loops, MI waveguides have also been formed as planar structures [12] and thin-film cables [13]. The latter have potential applications as patient-safe cable in magnetic resonance imaging $[14,15]$. Many MI devices have been proposed or demonstrated, including magnetic flux concentrators [16], delay lines [12], filters [17], directional couplers [4, 18], splitters [19], lenses for near-field imaging [20-22] and detectors for magnetic resonance imaging [23]. Parametric amplification has been considered as a method of reducing propagation losses [24, 25].

Despite this effort, losses per metre have historically been high $(150 \mathrm{~dB} / \mathrm{m}$ in [1] and 50 $\mathrm{dB} / \mathrm{m}$ in [7]), propagation distances have been limited, and end-reflections have mainly been ignored. However, the demonstration of losses of $\approx 2.5 \mathrm{~dB} / \mathrm{m}$ in thin-film cables [13] has re-affirmed the need for an effective transducer for coupling to a conventional transmission line or for termination. Without such a transducer, it will be difficult to construct any high-performance MI system. Although multi-element absorbers have been proposed [26], these do not solve the coupling problem. In this paper, we consider 
possibilities for inductive coupling to a MI waveguide, and demonstrate a simple broadband transducer that can be constructed from passive components. The theory of matching is described in Section 2, transducer optimisation is explored in more detail in Section 3, experimental verification is presented in Section 4, and conclusions are drawn in Section 5. 


\section{Matching to magneto-inductive waveguides}

We first consider the principle of matching a magneto-inductive waveguide to a real load. Figure 1a shows the equivalent circuit of an ideal, loss-less guide, formed from a set of L$\mathrm{C}$ resonators coupled to their nearest neighbours by a mutual inductance $\mathrm{M}$. Away from any termination $(n<0)$ the equation governing the current $I_{n}$ in the $n^{\text {th }}$ element at angular frequency $\omega$ can be found from Kirchhoff's voltage law as [1]:

$$
\{j \omega L+1 / j \omega C\} I_{n}+j \omega M\left\{I_{n-1}+I_{n+1}\right\}=0
$$

A solution can be found by assuming that $I_{n}$ is the travelling wave $I_{n}=I_{0} \exp ( \pm j n k a)$, where $\mathrm{I}_{0}$ is the wave amplitude, $\mathrm{ka}$ is the phase shift per element, $\mathrm{k}$ is the propagation constant and a is the period. Substitution into Equation 1 yields the well-known dispersion equation:

$$
\left(1-\omega_{0}{ }^{2} / \omega^{2}\right)+\kappa \cos (\mathrm{ka})=0
$$

Here $\omega_{0}{ }^{2}=1 / \mathrm{LC}$ is the angular resonant frequency and $\kappa=2 \mathrm{M} / \mathrm{L}$ the coupling coefficient. For positive $\kappa$, propagation can only take place for $\omega / \omega_{0}$ between $1 / \sqrt{ }(1+\kappa)$ and $1 / \sqrt{ }(1-$ $\kappa)$. The effect of loss may be modelled by repeating the analysis, assuming the presence of a resistor $\mathrm{R}$ in each resonant loop [1]. If this is done, the dispersion equation is modified to $\left(1-\omega_{0}{ }^{2} / \omega^{2}-\mathrm{j} / \mathrm{Q}\right)+\kappa \cos (\mathrm{ka})=0$, where $\mathrm{Q}=\omega \mathrm{L} / \mathrm{R}$ is the $\mathrm{Q}$-factor. Assuming a complex-valued propagation constant $\mathrm{ka}=\mathrm{k}$ 'a $-\mathrm{jk}$ ' $\mathrm{a}$, and further assuming that $\mathrm{k}$ ' $\mathrm{a}$ is 
small, it can be shown that k' $\mathrm{a}=1 /\left\{\kappa \mathrm{Q} \sin \left(\mathrm{k}^{\prime} \mathrm{a}\right)\right\}$. Losses are lowest at mid-band, and strong coupling and a high Q-factor are required for low loss.

In [2], it was shown that a non-reflective termination is formed by inserting an impedance $\mathrm{Z}_{0}$ into the last $\left(0^{\text {th }}\right)$ element of the waveguide, where $\mathrm{Z}_{0}$ is given by:

$$
\mathrm{Z}_{0}=\mathrm{j} \omega \mathrm{M} \exp (-\mathrm{jka})
$$

At mid-band, when $\omega=\omega_{0}$ and $\mathrm{ka}=\pi / 2, \mathrm{Z}_{0}$ reduces to the real value $\mathrm{Z}_{0 \mathrm{M}}=\omega_{0} \mathrm{M}$. Thus, in principle it should be possible to couple a magneto-inductive waveguide to a conventional transmission line (which has real impedance) if $\mathrm{M}$ is appropriately chosen. Unfortunately, $\mathrm{Z}_{0}$ is complex away from the band centre, and moreover as expressed here is a function of both ka and $\omega$. Consequently, Equation 3 has so far represented a mathematical contrivance, rather than an element that can be realised. Further development of MI systems clearly requires simple circuits that can approximate this impedance.

In a search for suitable circuits we consider the termination in Figure 1b. Here the final element of a MI waveguide is coupled via a mutual inductance $\mathrm{M}^{\prime}$ to a loop containing an inductance L', a capacitance C' and a real load $\mathrm{R}_{\mathrm{L}}$, which will typically represent a $50 \Omega$ system. The loop is resonant at an angular frequency $\omega_{0}{ }^{\prime}=1 / \mathrm{L}^{\prime} \mathrm{C}^{\prime}$, but may be considered non-resonant when $\omega_{0}{ }^{\prime}$ is zero. For the final elements, the circuit equations are: 


$$
\begin{gathered}
\{j \omega L+1 / j \omega C\} I_{0}+j \omega \mathrm{MI}_{-1}+j \omega M^{\prime} I_{L}=0 \\
\left\{R_{L}+j\left(\omega L^{\prime}-1 / \omega C^{\prime}\right)\right\} I_{L}+j \omega M^{\prime} I_{0}=0
\end{gathered}
$$

Combining Equations 4, we obtain for the $0^{\text {th }}$ element:

$$
\left\{j \omega \mathrm{L}+1 / j \omega \mathrm{C}+\mathrm{Z}_{\mathrm{L}}\right\} \mathrm{I}_{0}+\mathrm{j} \omega \mathrm{MI}_{-1}=0
$$

Here $\mathrm{Z}_{\mathrm{L}}$ is a load that has effectively been inserted into the $0^{\text {th }}$ element, given by:

$$
\mathrm{Z}_{\mathrm{L}}=\omega^{2} \mathrm{M}^{, 2} /\left\{\mathrm{R}_{\mathrm{L}}+\mathrm{j} \omega \mathrm{L}^{\prime}\left(1-\omega_{0}, 2 / \omega^{2}\right)\right\}
$$

We may evaluate the performance of $\mathrm{Z}_{\mathrm{L}}$ as a termination by considering the reflection of current waves as shown in Figure 1c. Assuming solutions of Equations 1 and 5 as the sum of incident and reflected waves, i.e. as $I_{n}=I_{I} \exp (-j n k a)+I_{R} \exp (+j k a)$, substituting into Equation 5 and using Equation 2 we can obtain the reflection coefficient $\Gamma=I_{R} / I_{I}$ as:

$$
\Gamma=-\left\{Z_{L}-Z_{0}\right\} /\left\{Z_{L}+Z_{0} *\right\}
$$

Equation 7 is similar to the reflection coefficient for current waves obtained when a conventional transmission line is terminated with a load. However, due to the presence of a complex conjugate term, it is clearly not identical. 
Since the current in element zero, which passes through the effective load, is $I_{0}=I_{I}+I_{R}$, we can also define a transmission coefficient as $\mathrm{T}=\mathrm{I}_{0} / \mathrm{I}_{\mathrm{I}}=(1+\Gamma)$, or:

$$
\mathrm{T}=2 \operatorname{Re}\left(\mathrm{Z}_{0}\right) /\left\{\mathrm{Z}_{\mathrm{L}}+\mathrm{Z}_{0} *\right\}
$$

Equation 8 is again similar, but not identical to the conventional transmission coefficient, due to the presence of a real operator. It is simple to show that $\Gamma$ and $T$ satisfy the power conservation relation:

$$
\Gamma \Gamma^{*}+\mathrm{TT}^{*} \operatorname{Re}\left(\mathrm{Z}_{\mathrm{L}}\right) / \operatorname{Re}\left(\mathrm{Z}_{0}\right)=1
$$

Once again, Equation 9 is similar to the corresponding result for real-valued systems.

Different terminations can be compared by plotting the scattering parameter $S_{11}$ in $d B$, as:

$$
S_{11} \approx 10 \log _{10}\left\{|\Gamma|^{2}\right\}
$$

Equation 7 implies that $\mathrm{Z}_{\mathrm{L}}$ should be chosen to approximate $\mathrm{Z}_{0}$ as far as possible. We now compare a number of possibilities, assuming for simplicity that $\mathrm{Z}_{0 \mathrm{M}}=\mathrm{R}_{\mathrm{L}}$ and that $\mathrm{M}^{\prime}$ $=\mathrm{M}$.

Figure 2 shows the frequency variation of $S_{11}$ obtained in a non-resonant transducer for different values of L'/L, calculated assuming the typical coupling coefficient $\kappa=0.6$. 
Propagation can take place over the band $0.79 \leq \omega / \omega_{0} \leq 1.58$, and $S_{11}$ rises to $0 \mathrm{~dB}$ at the band edges. Within the band, $S_{11}$ reduces somewhat. However, when $L^{\prime} / L=1$, the reflection coefficient is generally high and the transducer is correspondingly ineffective. As L'/L reduces, a deeper and deeper minimum in $S_{11}$ develops, and gradually shifts towards $\omega / \omega_{0}=1$. These results imply that the performance of the transducer improves as $\mathrm{L}^{\prime} / \mathrm{L}$ reduces, and that the best result is obtained when only a small reactance is inserted into the final loop. However, the best that can be achieved still only represents a narrowband impedance match. Furthermore, the minimum in $S_{11}$ is relatively high $(-35 \mathrm{~dB})$, and even this result is only obtained for very small values of $L^{\prime} / L$, when it may be difficult to maintain $\mathrm{M}^{\prime} / \mathrm{M}=1$.

The inserted reactance can clearly be cancelled more effectively if the transducer is made resonant. Figure 3 shows the corresponding variation of $S_{11}$ obtained for a resonant transducer, calculated assuming that $\kappa=0.6$ and that $L^{\prime}$ and $C^{\prime}$ are chosen so that $\omega_{0}{ }^{\prime}=$ $\omega_{0}$. Results are again shown for different values of $L^{\prime} / L$. The most obvious choice of $L^{\prime} / L$ $=1$ provides a complete null in reflectivity when $\omega / \omega_{0}=1$. However, this result again only represents a narrow-band match. In contrast, the less obvious choice of $L^{\prime} / L=0.5$ provides two nulls in reflectivity, the first again being at $\omega / \omega_{0}=1$. Because the nulls are widely separated, low reflectivity is obtained over a wide band. For example, $S_{11}$ is less than $-20 \mathrm{~dB}$ for $65 \%$ of the pass-band, and less than $-30 \mathrm{~dB}$ for $45 \%$. This form of transducer is extremely effective. Importantly, it can be realised very conveniently as shown in Figure 1d. Here each resonant element in a MI waveguide is now formed from two separate inductors of value $\mathrm{L} / 2$, and two capacitors of value $2 \mathrm{C}$. If half the final 
element is simply removed, the remainder may then be connected directly to a resistive load. This modification allows MI waveguides of arbitrary length to be terminated without the need for additional components. To realise such an arrangement, all that is required is to stagger the inductors and capacitors in each unit cell, so that the cell may be exactly bisected using a transverse cut. 


\section{3. $\quad$ Transducer optimisation}

We now consider the broadband resonant transducer in more detail. To understand how it achieves its effect, we introduce the normalised characteristic impedance $\mathrm{Z}_{0 \mathrm{~N}}=\mathrm{Z}_{0} / \omega_{0} \mathrm{M}$, obtained from Equation 3 as:

$$
\mathrm{Z}_{0 \mathrm{~N}}=\mathrm{w} /\{\sin (\mathrm{ka})-\mathrm{j} \cos (\mathrm{ka})\}
$$

Here $\mathrm{w}=\omega / \omega_{0}$ is a normalised frequency. Using the dispersion equation (2), $\mathrm{Z}_{0 \mathrm{~N}}$ may be written alternatively as:

$$
\mathrm{Z}_{0 \mathrm{~N}}=\mathrm{w} /\left\{\sqrt{ }\left[1-\left(1-1 / \mathrm{w}^{2}\right)^{2} / \mathrm{\kappa}^{2}\right]+\mathrm{j}\left(1-1 / \mathrm{w}^{2}\right) / \kappa\right\}
$$

In this form, $\mathrm{Z}_{0 \mathrm{~N}}$ is clearly a function only of $\mathrm{w}$. Consequently it may be compared directly with the corresponding normalised load impedance $\mathrm{Z}_{\mathrm{LN}} / \omega_{0} \mathrm{M}$, which may be written as:

$$
Z_{\mathrm{LN}}=w /\left\{\rho / w \mu^{2}+j\left(2 \lambda / \kappa \mu^{2}\right)\left(1-\eta^{2} / w^{2}\right)\right\}
$$

Here we have introduced four normalised variables. The first, $\rho=\mathrm{R}_{\mathrm{L}} / \omega_{0} \mathrm{M}$, is the ratio of the $\mathrm{R}_{\mathrm{L}}$ to the mid-band impedance of the MI waveguide. The second, $\lambda=\mathrm{L}$ '/L, is the ratio of the self-inductances in the transducer and the guide, the third, $\mu=\mathrm{M}^{\prime} / \mathrm{M}$, is the ratio of mutual inductances, and the fourth, $\eta=\omega_{0}{ }^{\prime} / \omega_{0}$, is the ratio of resonant frequencies. 
Clearly, reflectivity will be low if the real and imaginary parts of $\mathrm{Z}_{0 \mathrm{~N}}$ and $\mathrm{Z}_{\mathrm{LN}}$ are similar, or alternatively if the real and imaginary parts of the normalised admittances $Y_{0 N}=1 / Z_{0 N}$ and $\mathrm{Y}_{\mathrm{LN}}=1 / \mathrm{Z}_{\mathrm{LN}}$ correspond. Considering first the imaginary parts, $\operatorname{Im}\left(\mathrm{Y}_{0 \mathrm{~N}}\right)$ can be made equal to $\operatorname{Im}\left(\mathrm{Y}_{\mathrm{LN}}\right)$ for all $\omega$ if $\eta=1$ and $\lambda=\mu^{2} / 2$. If $\mu=1$, as previously assumed, a complete match in admittance can therefore be obtained if the transducer is resonant at $\omega_{0}$ and the inductance L' of the transducer is half that of the resonant elements forming the guide.

Considering now the real parts, and assuming that the transducer is correctly resonant, $\operatorname{Re}\left(\mathrm{Y}_{0 \mathrm{~N}}\right)$ can be made equal to $\operatorname{Re}\left(\mathrm{Y}_{\mathrm{LN}}\right)$ if:

$$
\rho / w \mu^{2}=\sqrt{ }\left[1-\left(1-1 / w^{2}\right)^{2} / \kappa^{2}\right]
$$

Equation 14 is actually a quadratic equation, which can be expanded as:

$$
\mathrm{w}^{4}\left\{\kappa^{2}-1\right\}+\mathrm{w}^{2}\left\{2-\alpha^{2} \kappa^{2}\right\}-1=0
$$

Here, $\alpha=\rho / \mu^{2}$. When $\alpha=1$, a condition that can be achieved by taking $M^{\prime}=M$ and $\omega_{0} M$ $=\mathrm{R}_{\mathrm{L}}$, Equation 15 has the simple solutions of $\mathrm{w}=1$ and $\mathrm{w}=1 / \sqrt{ }\left(1-\kappa^{2}\right)$. Both lie in the propagating band. These results imply that if $\omega_{0} M=R_{L}$, and if the transducer is made resonant at $\omega_{0}$ using an inductance $L^{\prime}=L / 2$ (which requires a capacitance $C^{\prime}=2 C$ ), the imaginary parts of $\mathrm{Y}_{\mathrm{L}}$ and $\mathrm{Y}_{0}$ can be made equal across the band and the real parts at two 
discrete frequencies. Consequently, $\mathrm{Z}_{0}$ and $\mathrm{Z}_{\mathrm{L}}$ can be equalised at the same two frequencies, and at these points there can be no reflection. These results are confirmed in Figure 4, which shows the frequency variations of the real and imaginary parts of $Z_{0 N}$ and $\mathrm{Z}_{\mathrm{LN}}$, calculated assuming $\kappa=0.6$. Matching is achieved when $\mathrm{w}=\omega / \omega_{0}=1$ and $\omega / \omega_{0}=$ $1 / \sqrt{ }\left(1-0.6^{2}\right)=1.25$, the points at which nulls in reflectivity are seen in Figure 3. Broadband operation then follows from the existence of these two separate nulls.

A broadband transducer can still be constructed if $\alpha \neq 1$ (for example, if $M^{\prime}=M$ but $\omega_{0} M$ $\neq \mathrm{R}_{\mathrm{L}}$ ). Figure 5 shows the frequency variation of the scattering parameter $\mathrm{S}_{11}$ for resonant transducers, calculated assuming $\kappa=0.6$, and $L^{\prime} / L=0.5$ and assuming different values of $\alpha$. For $\alpha<1$, there are again two nulls in reflectivity, which move further apart as $\alpha$ reduces. For $\alpha>1$, there is only a single minimum, not a null. However, in each of the cases shown, the return is generally low over a wide spectral range. These results suggest that the broadband resonant transducer will give reasonable performance even when the mid-band impedance of the MI waveguide is slightly mismatched from $R_{L}$. Finally, it is simple to show that the two nulls in reflectivity just merge together when the roots of Equation 15 are repeated. This occurs when 


$$
\left(2-\alpha^{2} \kappa^{2}\right)^{2}=4\left(1-\kappa^{2}\right)
$$

Or when:

$$
\alpha^{4} \kappa^{2}-4 \alpha^{2}+4=0
$$

Equation 17 has the solutions $\left.\alpha=(\sqrt{ } 2 / \kappa)\left\{1 \pm \sqrt{ }\left(1-\kappa^{2}\right)\right]\right\}^{1 / 2}$. For $\kappa=0.6$, for example, the solutions are $\alpha=3.162$ and $\alpha=1.054$. The latter value is midway between $\alpha=1$ and $\alpha=$ 1.1 , and generates a variation in reflectivity with a single null. 


\section{Experimental verification}

Experimental confirmation of the theory of the previous section was provided using thinfilm magneto-inductive cables formed by double-sided patterning of copper-clad polyimide, as described in [13]. Figure 6a shows the physical arrangement and key dimensions, and Figure $6 \mathrm{~b}$ a short section of cable. Each resonant element is formed from two inductors and two capacitors. The inductors are single-turn loops of inductance L/2, located on either side of a thin substrate, while the capacitors are parallel-plate components of capacitance $2 \mathrm{C}$, which use the substrate as a dielectric interlayer. This arrangement approximates that of Figure 1d, although it does not allow a resonant termination to be formed by cutting the cable. Instead, it provides non-resonant terminations of inductance $\mathrm{L}^{\prime}=\mathrm{L} / 2$, which may be made resonant with additional capacitors. Since the mutual inductances $M$ and $M^{\prime}$ are the same, this arrangement has normalised parameter values $\lambda=0.5$ and $\mu=1$.

Cables were fabricated in two metre lengths by the UK company Clarydon (Willenhall, West Midlands). The base material consisted of $25 \mu \mathrm{m}$ thick Kapton ${ }^{\circledR}$ carrying a $35 \mu \mathrm{m}$ thick layer of copper on either side. The copper was patterned by step-and-repeat lithographic exposure to a pair of one metre long photomasks, followed by wet etching. The photomasks contained a set of MI waveguides with different parameters. The overall width and length were taken as $\mathrm{w}=4.7 \mathrm{~mm}$ and $\mathrm{a}=100 \mathrm{~mm}$ throughout, so that a twometre length contained 19 resonant elements. The track width $t$, and the small gaps $\mathrm{g}_{\mathrm{C}}$ and $\mathrm{g}_{\mathrm{L}}$ between capacitor plates and between plates and tracks were all taken as $0.5 \mathrm{~mm}$. The 
main variables were the capacitor and inductor lengths $d_{C}$ and $d_{L}$, which were varied to obtain different properties. Of particular importance were the inductance $\mathrm{L}$, the capacitance $\mathrm{C}$, the mutual inductance $\mathrm{M}$ and the $\mathrm{Q}$-factor of the resonant elements. These parameters determine the resonant frequency $\mathrm{f}_{0}=\omega_{0} / 2 \pi$, the coupling coefficient $\kappa=$ $2 \mathrm{M} / \mathrm{L}$, the mid-band impedance $\mathrm{Z}_{0 \mathrm{M}}=\omega_{0} \mathrm{M}$ and an imaginary part of the propagation constant.

Electrical performance was evaluated using an Agilent E5061A Electronic Network Analyser (ENA). The inductance was determined by making the transducers resonant at low frequency with a known capacitor and measuring the resonant frequency with a weak inductive probe. The remaining parameters were estimated by attaching SMA-type endlaunch connectors as shown in Figure 6c, measuring transmission and reflection data, and fitting the data to a theoretical model. Recently, it has been shown that flexible magnetoinductive cable is very tolerant to bends, due to the extreme stability of the mutual inductance between adjacent elements [27]. Despite this, small reflections tend to mask the appearance of predicted nulls in $S_{11}$. The smallest overall reflections were therefore obtained with the MI waveguide held straight, using an additional co-axial cable to return the transmitted signal to the ENA.

The theoretical model includes propagation loss but ignores multiple reflections. In this case, the scattering parameters $\mathrm{S}_{11}$ and $\mathrm{S}_{21}$ for an $\mathrm{N}$-element MI waveguide connected to a source with real output impedance $\mathrm{R}_{\mathrm{L}}$ and a similar load are given approximately by: 


$$
\begin{gathered}
\mathrm{S}_{11} \approx 10 \log _{10}\left\{|\Gamma|^{2}\right\} \\
\mathrm{S}_{21} \approx 10 \log _{10}\left\{\left(1-|\Gamma|^{2}\right) \exp (-2 \mathrm{Nk}, \mathrm{a})\left(1-|\Gamma|^{2}\right)\right\}
\end{gathered}
$$

Figure 7 shows a comparison between experimental measurement of the frequency variations of $S_{11}$ and $S_{21}$ and the theory above, for a cable with $d_{C}=10 \mathrm{~mm}$ and $d_{L} \approx 90$ $\mathrm{mm}$. The experimental data show band-limited propagation between $70 \mathrm{MHz}$ and 160 MHz. Overall transmission is high, and $\mathrm{S}_{21}$ peaks at $-8 \mathrm{~dB}$ near $110 \mathrm{MHz}$. Oscillations in transmission and reflection are due to multiple reflections, and suggest low propagation loss. However, the return is high, and the minimum value of $S_{11}$ is only $\approx-7 \mathrm{~dB}$. These results confirm the poor performance of non-resonant transducers. The agreement between theory and experiment is clearly good, apart from the inability of the simple theory used here to model multiple reflections and a small discrepancy in $S_{21}$ at high frequency.

The combination of direct measurement and matching to theory allowed deduction of the following parameter values. The inductance was estimated as $\mathrm{L}=241 \mathrm{nH}$, the resonant frequency as $\mathrm{f}_{0}=95 \mathrm{MHz}$, the capacitance as $\mathrm{C}=11.6 \mathrm{pF}$, the coupling coefficient as $\kappa=$ 0.675 , the mid-band impedance as $\mathrm{Z}_{0 \mathrm{M}}=48.6 \Omega$ and the $\mathrm{Q}$-factor as $\mathrm{Q}=48$ (which in turn implies a mid-band propagation loss of $0.27 \mathrm{~dB} / \mathrm{m}$ ). The mid-band impedance is clearly close to $50 \Omega$, and gives a value of $\rho=R_{L} / Z_{0} M=1.028$. 
The terminations were made resonant using surface mount capacitors, using the optimum capacitance value of $2 \times 11.6 \approx 23 \mathrm{pF}$. Electrical performance was then re-measured to give the results shown in Figure 8. Here, peak transmission has now increased to $-5.2 \mathrm{~dB}$, and the oscillations due to multiple reflections have largely disappeared. The return has significantly reduced, and $\mathrm{S}_{11}$ is below $-25 \mathrm{~dB}$ for much of the band. The data are again compared with theory, this time assuming a resonant termination, and good agreement is again obtained. However, the predicted nulls in reflection cannot be seen in the experiment, presumably due to other small reflections from connections. These results confirm the improvement in performance offered by the optimum resonant termination. 


\section{5. $\quad$ Conclusions}

A simple inductive transducer for coupling a magneto-inductive waveguide to a real load has been introduced. A theory of reflection from lumped-element coupling transducers has been developed. It has been shown that zero reflection can be obtained at a single frequency if the (real-valued) mid-band impedance of the MI waveguide matches the load, and if the transducer is resonant at the same frequency as the resonant elements forming the guide. If in addition the inductance of the transducer is half that used in the resonant elements and the capacitance is correspondingly double, matching to the load can also be achieved at a second frequency. Since zero reflection is now obtained at two separate frequencies, low reflectivity can be obtained over a broad spectral range. The theory has been compared with experimental results obtained from thin-film magnetoinductive cable. Excellent agreement has been obtained, and the improvement in performance offered by the optimised resonant transducer has been confirmed by comparison with a non-resonant equivalent.

Other more complicated transducer designs can doubtless be developed to achieve improved broadband performance. However, the simple design presented here has the important advantage that a transducer with exactly the required properties can be obtained from the waveguide itself, if the resonant elements are formed using pairs of inductors and capacitors in series, rather than single components. As a result, a connection between a MI waveguide and a system with real impedance may be obtained simply by splicing, exactly as is done for other cable types such as co-axial cable. The demonstration of a 
simple arrangement for coupling should greatly increase potential applications for MI waveguides. 


\section{6. $\quad \underline{\text { References }}$}

1. Shamonina E., Kalinin V.A., Ringhofer K.H., Solymar L. "Magneto-inductive waveguide" Elect. Lett. 르, 371-373 (2002)

2. Shamonina E., Kalinin V.A., Ringhofer K.H., Solymar L. "Magnetoinductive waves in one, two and three dimensions" J. Appl. Phys. $\underline{92}$, 6252-6261 (2002)

3. Wiltshire M.C.K., Shamonina E., Young I.R., Solymar L. "Dispersion characteristics of magneto-inductive waves: comparison between theory and experiment" Elect. Lett. $\underline{39}, 215-217(2003)$

4. Shamonina E., Solymar L. "Magneto-inductive waves supported by metamaterial elements: components for a one-dimensional waveguide" J. Phys. D. Appl. Phys. $\underline{37}$, $362-367$ (2004)

5. Maslovski S., Ikonen P., Kolmakov I., Tretyakov S. “Artificial magnetic materials based on the new magnetic particle: metasolenoid" PIER 54, 61-81 (2005)

6. Jylhä L., Maslovski S., Tretyakov “Traveling waves along the metasolenoid” Progress in Electromagnetics Research Symposium, Cambridge, MA, March 26-29 (2009)

7. Syms R.R.A., Young I.R., Solymar L. "Low loss magneto-inductive waveguides" J. Phys. D. Appl. Phys. $\underline{39}$, 3945-3951 (2006)

8. Syms R.R.A., Sydoruk O., Shamonina E., Solymar L. "Higher order interactions in magneto-inductive waveguides" Metamaterials 1, 44-51 (2007)

9. Radkovskaya A., Sydoruk O., Shamonin M., Shamonina E., Stevens C.J., Faulkner G., Edwards D.J., Solymar L. "Experimental study of a bi-periodic magnetoinductive waveguide: comparison with theory" IET Micr. Antennas Propag. 1, 80-83 (2007) 
10. Syms R.R.A., Shamonina E., Kalinin V., Solymar L. "A theory of metamaterials based on periodically loaded transmission lines: interaction between magneto-inductive and electro-magnetic waves" J. Appl. Phys. 97, Art. \# 064909 (2005)

11. Radkovskaya A., Shamonin M., Stevens C.J., Faulkner G., Edwards D.J., Shamonina E., Solymar L. "An experimental study of the properties of magnetoinductive waves in the presence of retardation" J. Magnetism and Magnetic Mats $\underline{300}$, 29-32 (2006)

12. Freire M.J., Marques R., Medina F., Laso M.A.G., Martin F. "Planar magnetoinductive wave transducers: Theory and applications" Appl. Phys. Letts. $\underline{85}, 4439$ $4441(2004)$

13. Syms R.R.A., Solymar L., Young I.R., Floume T. "Thin-film magneto-inductive cable” J. Phys. D. Appl. Phys., submitted for publication

14. Syms R.R.A., Solymar L., Young I.R. "Periodic analysis of MR-safe transmission lines” IEEE J. Sel. Top. in Quant. Elect., DOI 10.11.09/JSTQE.2009.2032782 (2009)

15. Weiss S., Vernickel P., Schaeffter T., Schulz V., Gleich B. "Transmission line for improved RF safety of interventional devices” Mag. Res. Med. 54, 182-189 (2005)

16. Wiltshire M.C.K., Shamonina E., Young I.R., Solymar L. "Resonant magnetic concentrators” Progress in Electromagnetics Research Symposium, PIERS 2003, October 13-16, Honolulu, Hawaii, USA (2003)

17. Nefedov I.S., Tretyakov S.A. "On potential applications of metamaterials for the design of broadband phase shifters" Micr. Opt. Tech. Letts 145, 98-102 (2005)

18. Radkovskaya A., Sydoruk O., Shamonin M., Stevens C.J., Faulkner G., Edwards D.J., Shamonina E., Solymar L. "Transmission properties of two shifted magnetoinductive waveguides” Micr. Opt. Tech. Lett. $\underline{49}$, 1054-1058 (2007) 
19. Syms R.R.A., Shamonina A., Solymar L. "Magneto-inductive waveguide devices" IEE Proc. Micr. Ant. Propag. 153, 111-121 (2006)

20. Freire M.J., Marques R. "Planar magnetoinductive lens for three-dimensional subwavelength imaging” Appl. Phys. Letts. $\underline{86}$, art. 182505 (2005)

21. Sydoruk O., Shamonin M., Radkovskaya A., Zhuromskyy O., Shamonona E., Trautner R., Stevens C.J., Faulkner G., Edwards D.J., Solymar L. "Mechanism of subwavelength imaging with bilayered magnetic metamaterials: theory and experiment” J. Appl. Phys. 101, 073903 (2007)

22. Freire M.J., Marques R., Jelinek L. "Experimental demonstration of a $\mu=-1$ metamaterial lens for magnetic resonance imaging” Appl. Phys. Lett. 93, 231108 (2008)

23. Solymar L., Zhuromskyy O., Sydoruk O., Shamonina E., Young I.R., Syms R.R.A. "Rotational resonance of magnetoinductive waves: basic concept and application to

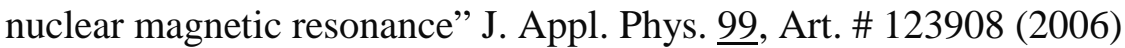

24. Sydoruk O., Shamonina E., Solymar L. "Parametric amplification in coupled magnetoinductive waveguides” J. Phys. D: Appl. Phys. 40, 6879-6887 (2007)

25. Syms R.R.A., Young I.R., Solymar L. "Three-frequency parametric amplification in magneto-inductive ring resonators" Metamaterials $\underline{2}$, 122-134 (2008)

26. Syms R.R.A., Solymar L., Shamonina E. “Absorbing terminations for magnetoinductive waveguides” IEE Proc. Micr. Antennas Propag. 152, 77-81 (2005)

27. Syms R.R.A., Solymar L. "Bends in magneto-inductive waveguides" Metamaterials, accepted for publication 


\section{7. $\quad \underline{\text { Figures }}$}

1. Magneto-inductive waveguide, with a) ideal termination, b) real termination c) equivalent termination and d) self-termination.

2. Frequency variation of the scattering parameter $S_{11}$ for non-resonant transducers calculated assuming $M^{\prime}=M, \kappa=0.6$ and different values of $L^{\prime} / L$.

3. Frequency variation of the scattering parameter $S_{11}$ for resonant transducers, calculated assuming $M^{\prime}=M, \kappa=0.6, \omega_{0}^{\prime}=\omega_{0}$ and different values of $L^{\prime} / L$.

4. Frequency variation of the real and imaginary parts of the normalised impedance $Z_{0 N}$ $=\mathrm{Z}_{0} / \omega_{0} \mathrm{M}$ (thin lines) and $\mathrm{Z}_{\mathrm{LN}}=\mathrm{Z}_{\mathrm{L}} / \omega_{0} \mathrm{M}$ (thick lines), calculated assuming $\mathrm{M}^{\prime}=\mathrm{M}, \kappa$ $=0.6, \omega_{0}{ }^{\prime}=\omega_{0}$ and $L^{\prime}=\mathrm{L} / 2$.

5. Frequency variation of the scattering parameter $S_{11}$ for resonant transducers, calculated assuming $M^{\prime}=M, \kappa=0.6, L^{\prime} / L=0.5$ and different values of $\alpha$.

6. a) Arrangement and b) experimental realisation of thin-film magneto-inductive cable; c) arrangement for resonant connection to conventional coaxial cable.

7. Frequency variation of $S_{11}$ and $S_{21}$ for thin-film magneto-inductive cable, with nonresonant transducers. Thick lines are experimental data and thin lines are theory.

8. Frequency variation of $S_{11}$ and $S_{21}$ for thin-film magneto-inductive cable, with resonant transducers. Thick lines are experimental data and thin lines are theory. 

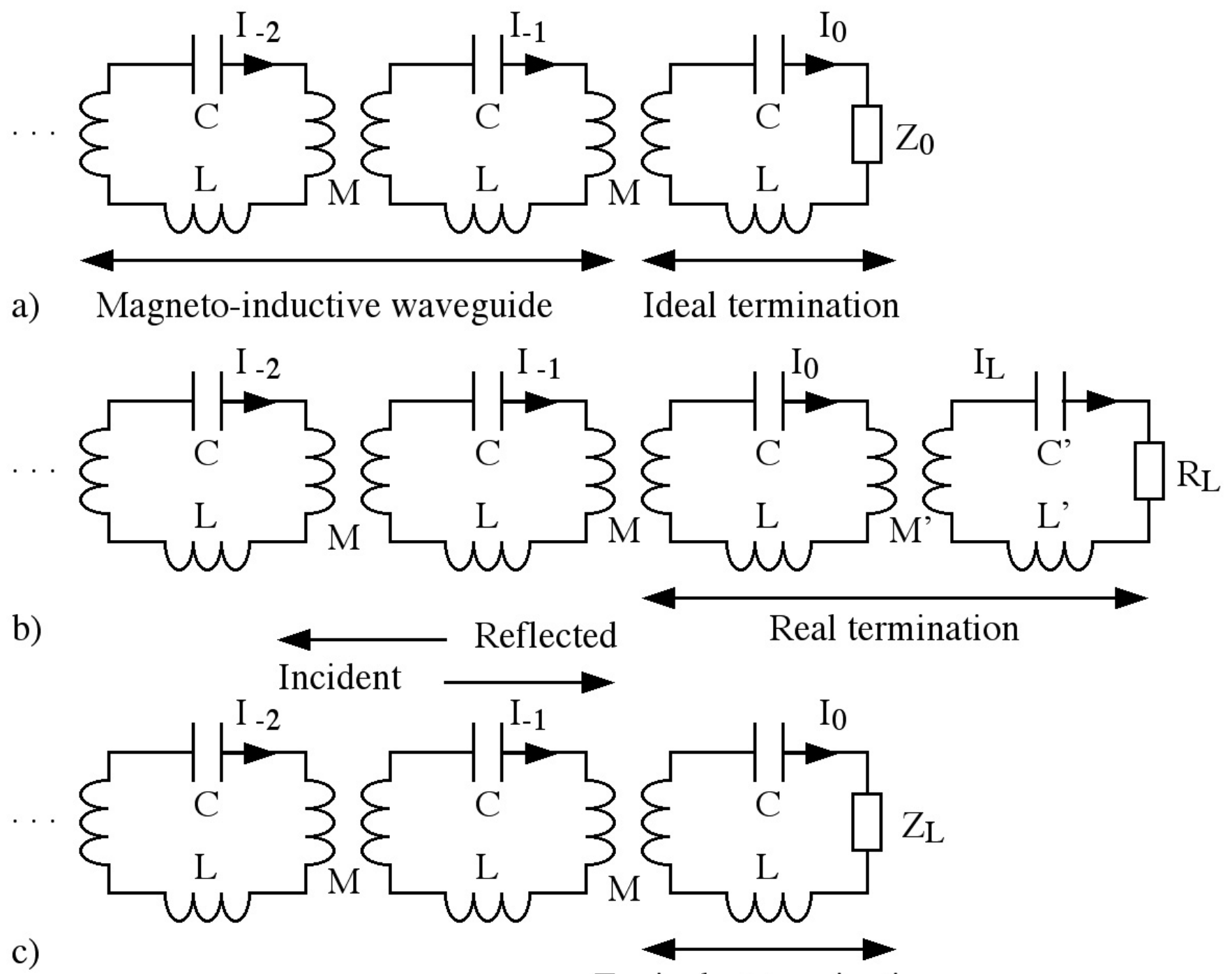

Equivalent termination

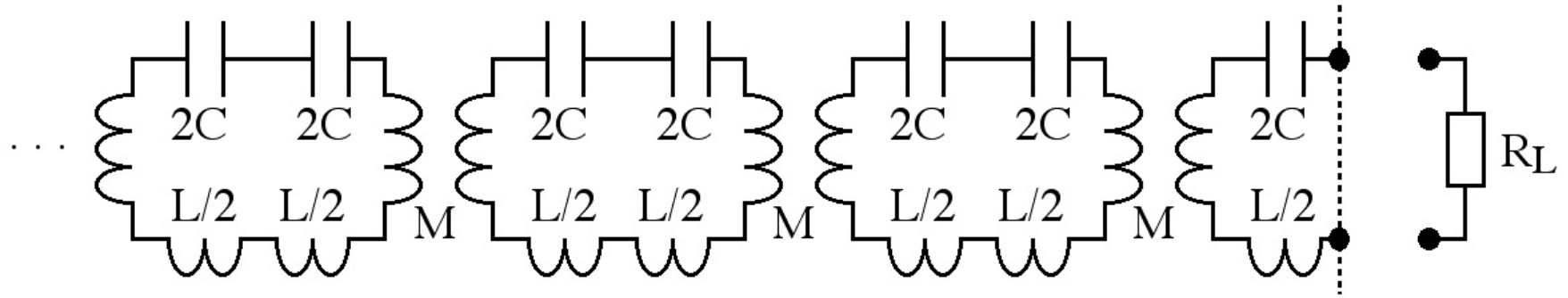

d)

Severed element

Figure 1 (Fig1.jpg) 


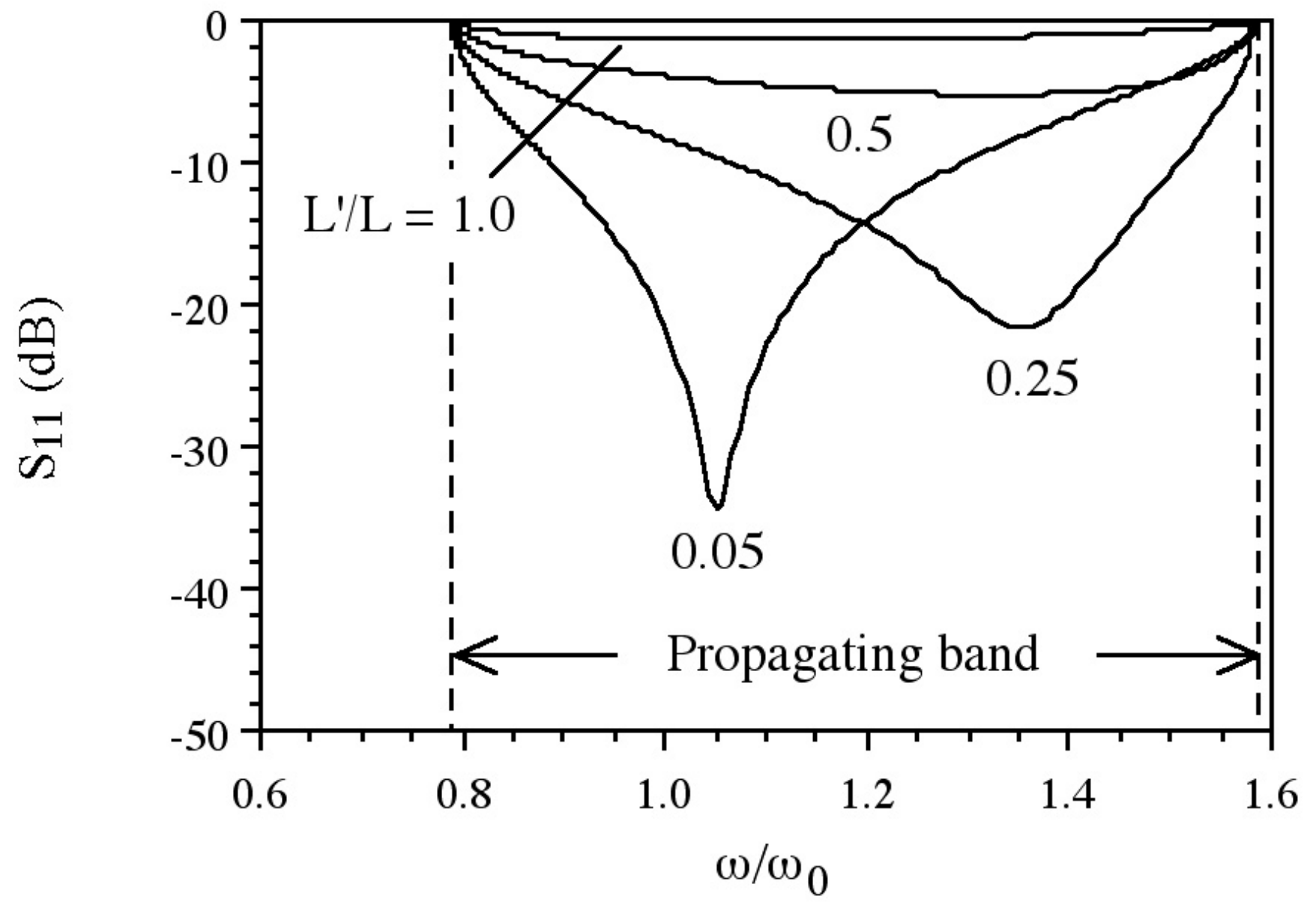

Figure 2 (Fig2.jpg) 


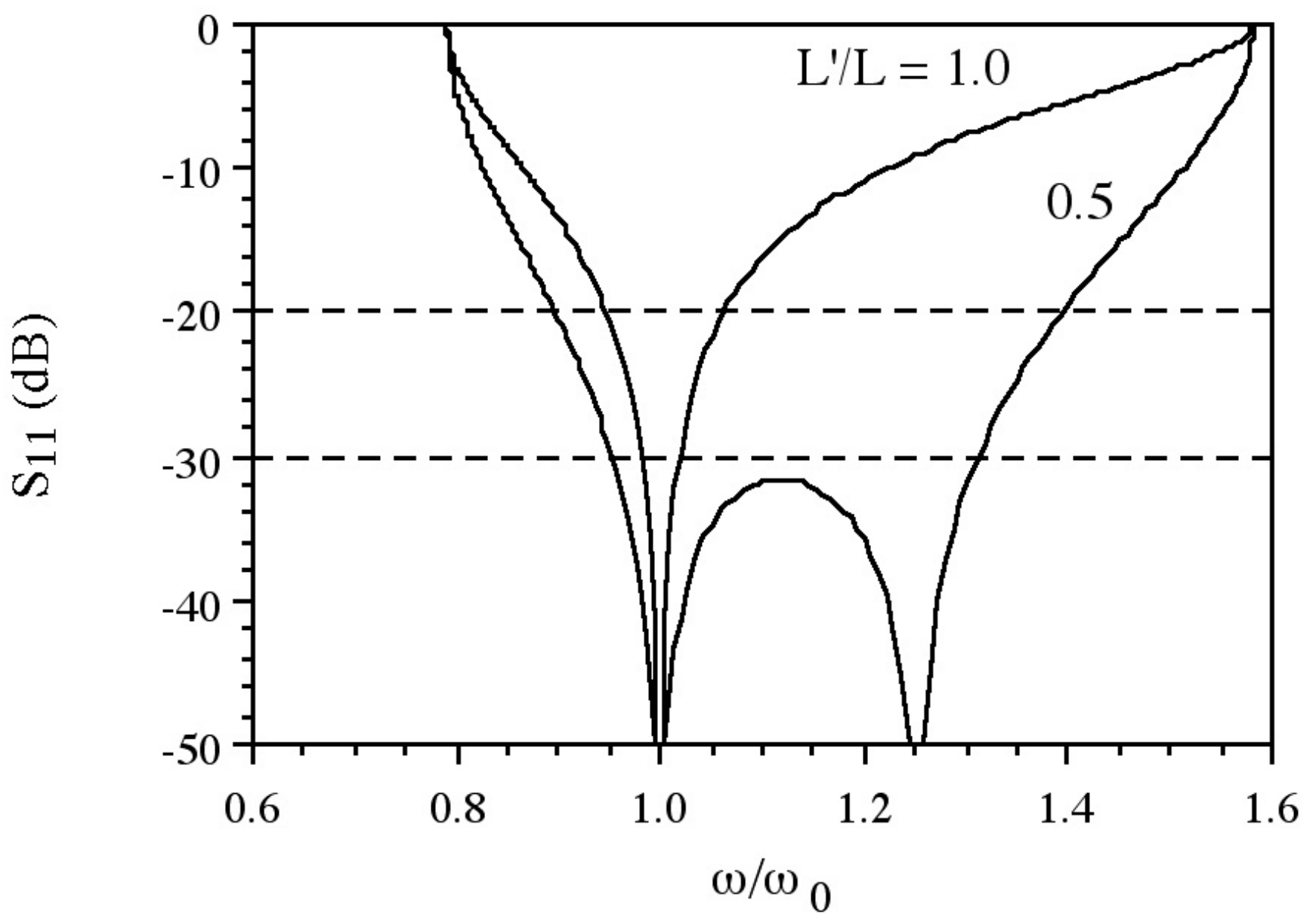

Figure 3 (Fig3.jpg) 


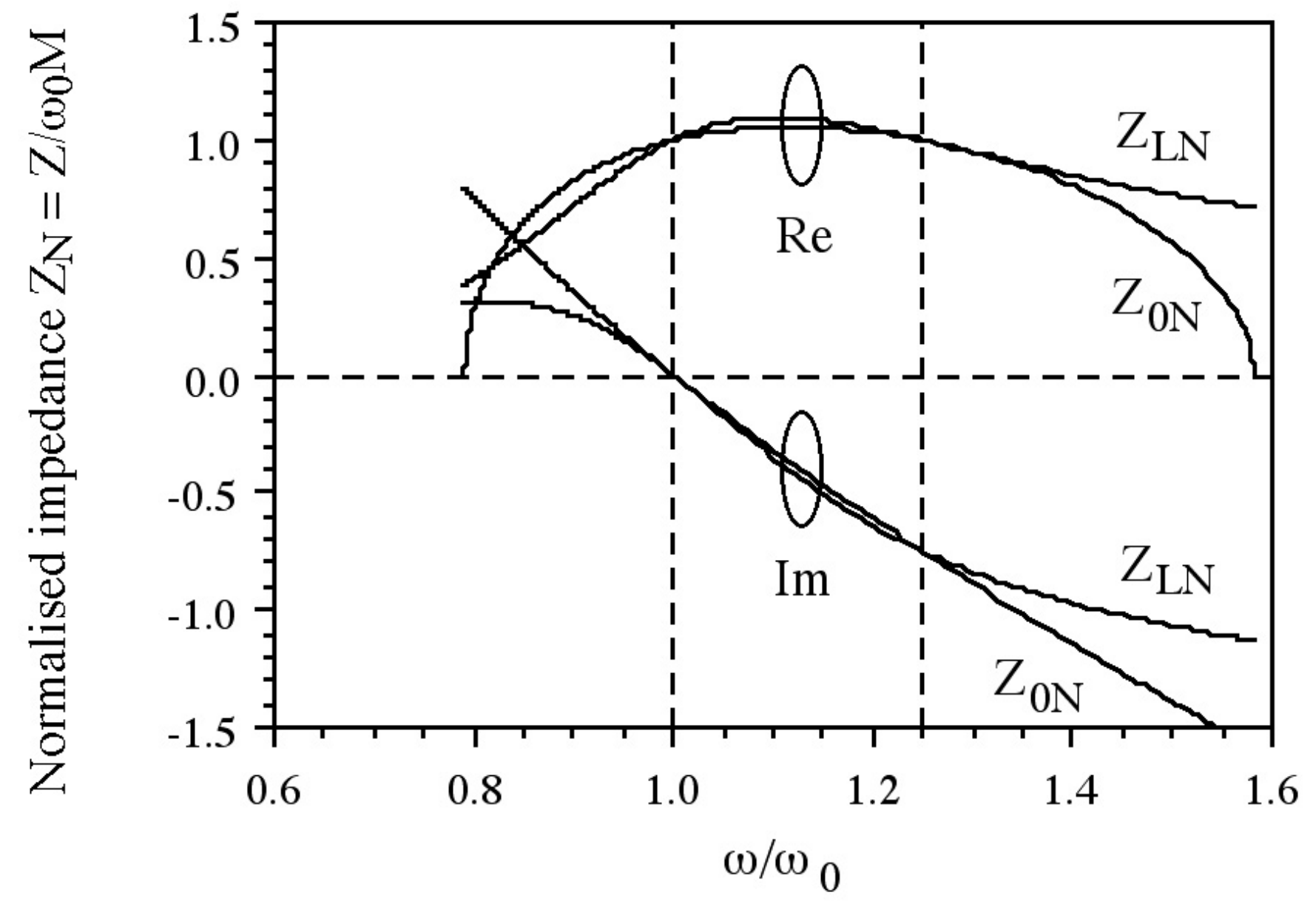

Figure 4 (Fig4.jpg) 


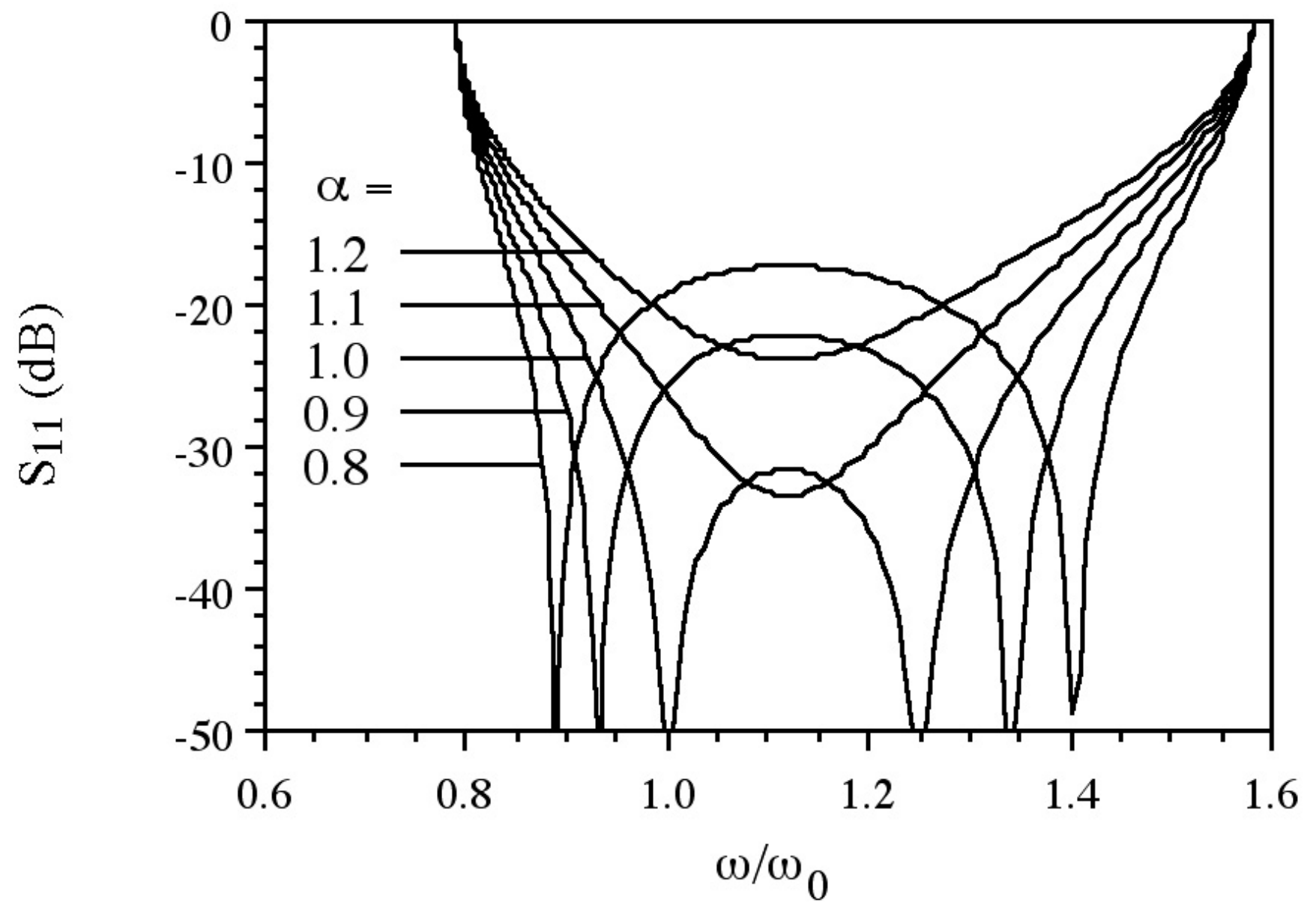

Figure 5 (Fig5.jpg) 


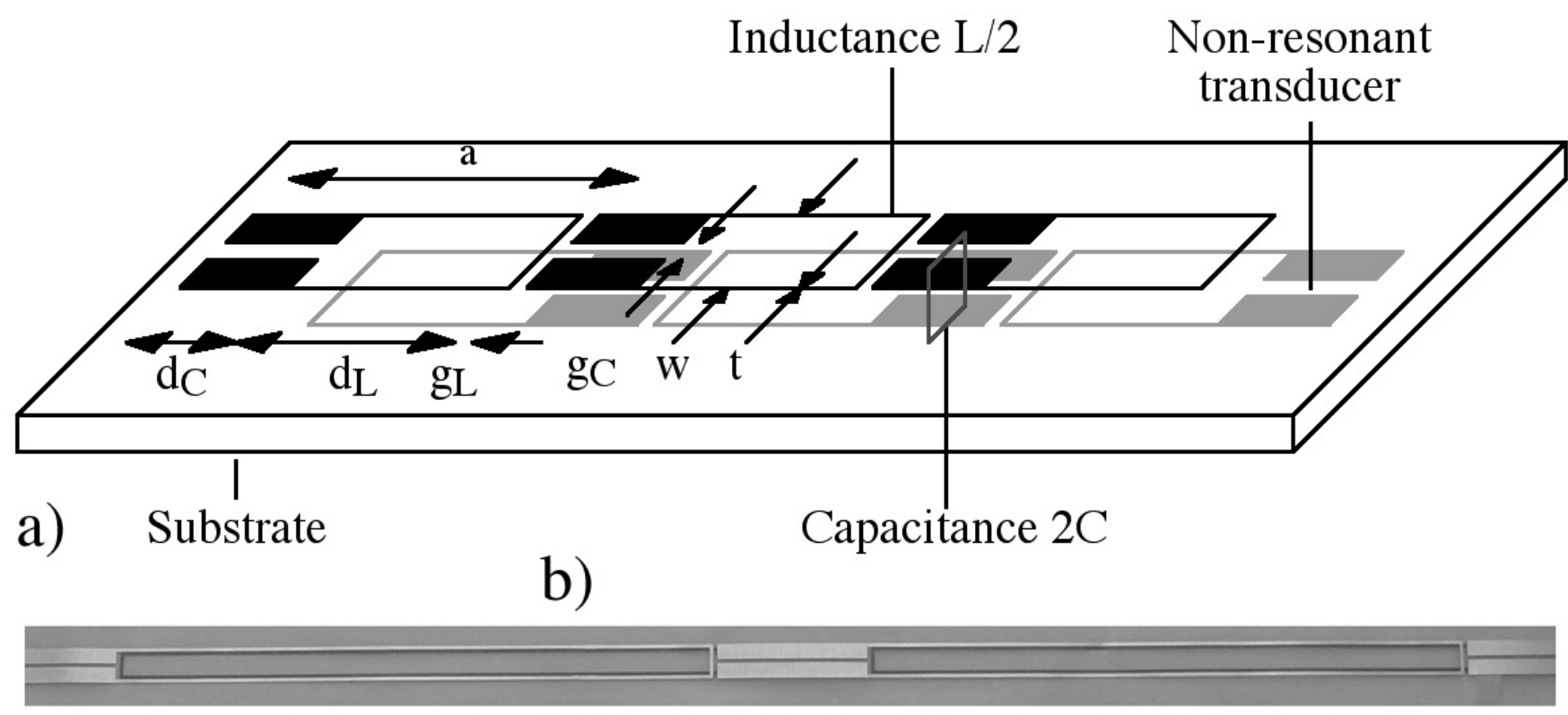

Figure 6 (Fig6.jpg) 


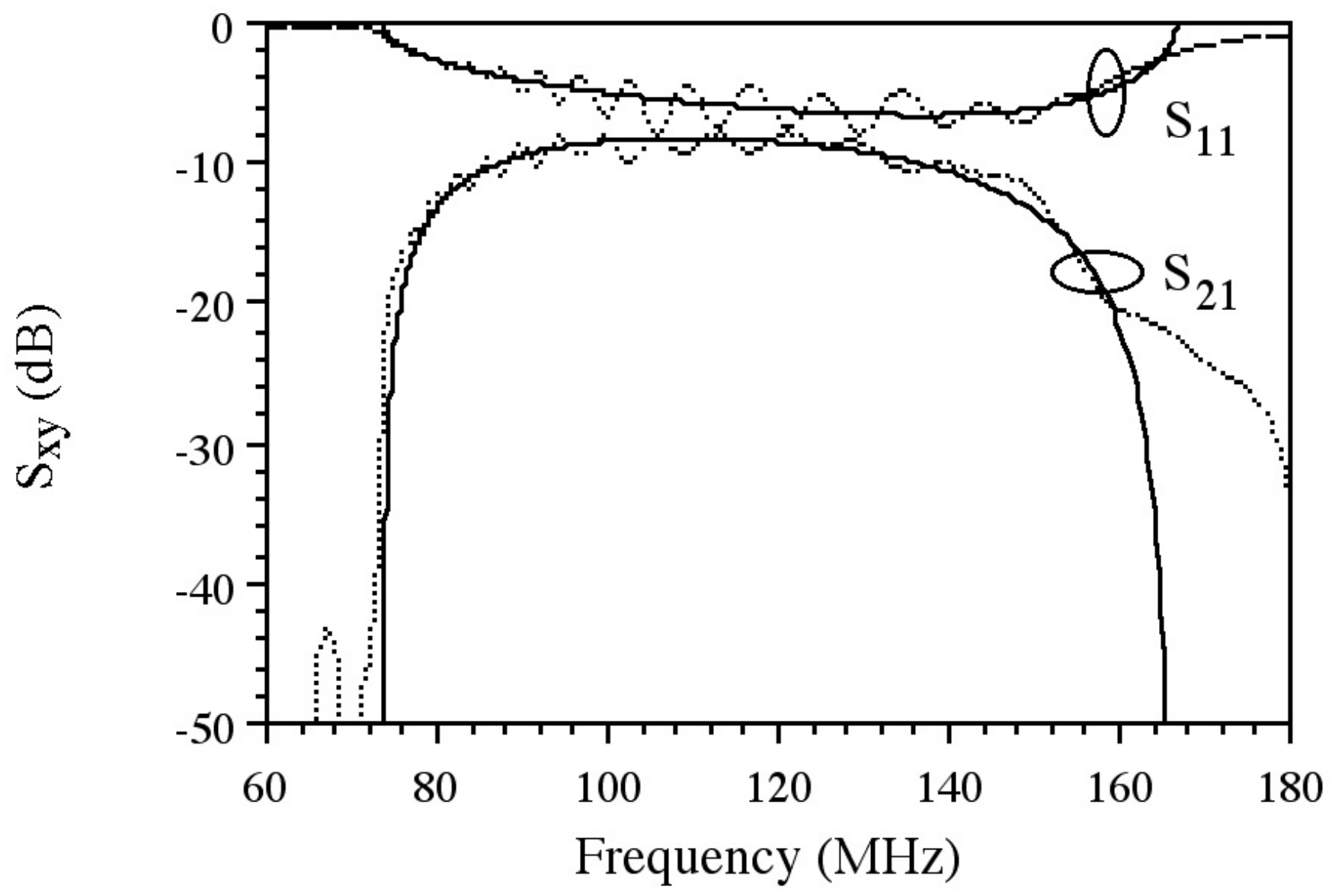

Figure 7 (Fig7.jpg) 


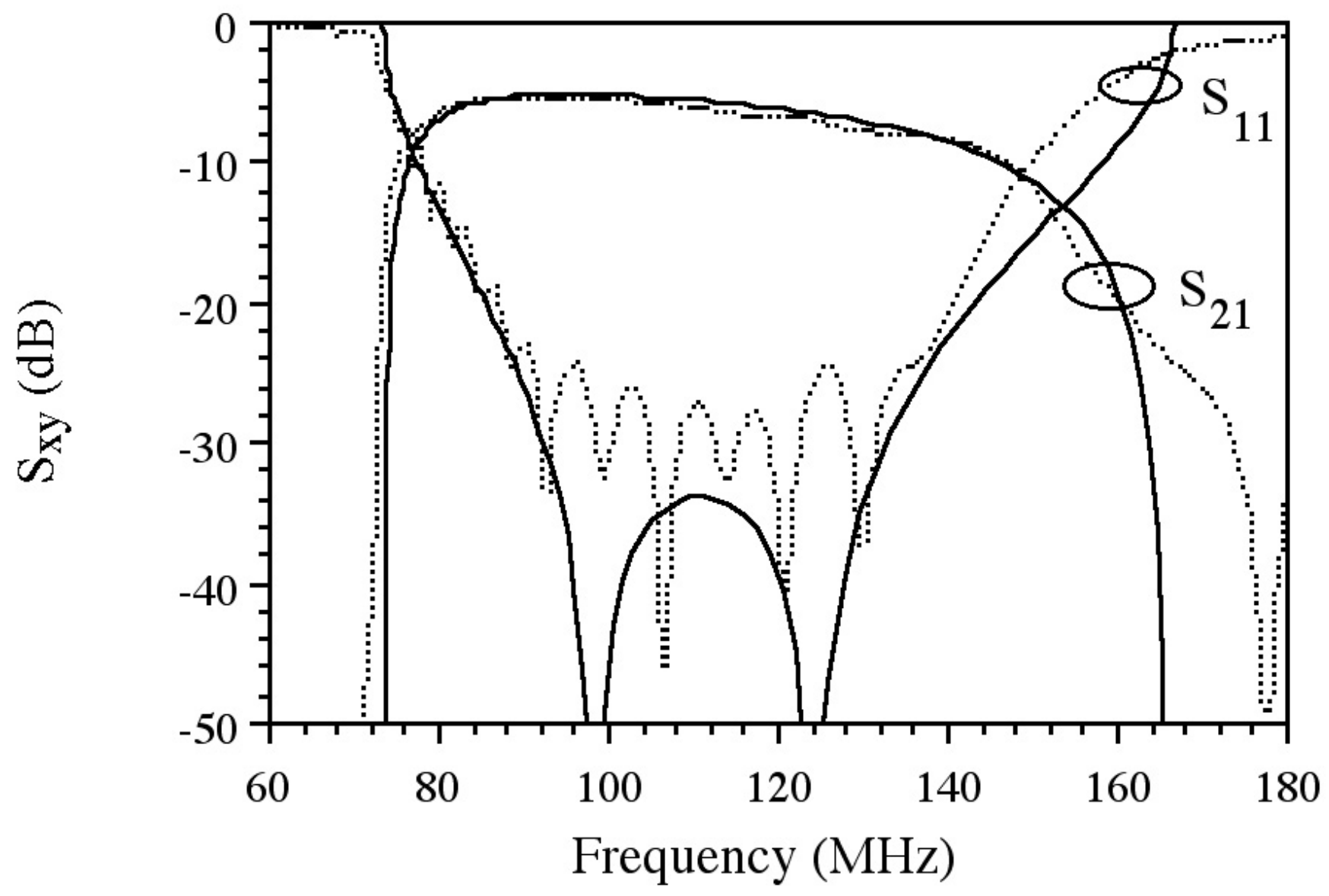

Figure 8 (Fig8.jpg) 